\title{
Do Bilingual Infants Possess Enhanced Cognitive Skills?
}

\author{
Angeline Sin Mei Tsui \\ [astsui@stanford.edu] \\ Department of Psychology \\ Stanford University
}

\author{
Christopher T. Fennell \\ [fennell@uottawa.ca] \\ School of Psychology \\ University of Ottawa
}

\begin{abstract}
Prior studies have reported that bilingualism enhances cognitive ability due to the regular conflict management of two language systems (Bialystok, 2015). Here, we explore whether infant bilingualism improves cognitive ability at 9.5 months. Twenty-four monolingual English and 23 bilingual French-English infants were first trained to predict a reward on the right based on a set of tone-shape rule structure (AAB pattern). Infants were later trained to predict a different reward on the left based on another set of new rule structure (ABB pattern). Correct anticipation of reward locations indicates successful learning. If bilingualism improves infants' cognitive skills, bilingual infants would be better at learning a new pattern-reward association. However, we did not find evidence that bilinguals looked at the correct location more than monolinguals or learned the new pattern-reward association faster. Thus, our results suggest bilingualism may not enhance cognitive ability at 9.5 months, as least using the current paradigm.
\end{abstract}

Keywords: infant bilingualism; cognitive ability; bilingual advantage

\section{Introduction}

Bilingual infants face key language learning challenges. For example, as their time listening to language input is divided between the two languages, they therefore hear less input from each language. In addition, bilingual infants often learn their languages in a more variable environment than monolinguals. It is not rare for bilingual infants to listen to parents mixing languages in one conversation (Byers-Heinlein, 2013) or to hear words form speakers with non-native accents (Bosch \& Ramon-Casas, 2011). Yet, bilingual infants reach basic language milestones at similar age as monolinguals, such as sensitivity to native sounds (Ferjan Ramirez et al., 2017) and basic word learning (for a review, see Fennell, Tsui, \& Hudon, 2016). This raises the possibility that bilingual infants may have better cognitive control processes because they are remarkably efficient in managing two different language systems.

A number of studies have suggested that bilingual experiences may enhance individuals' executive functioning (see Bialystok, Craik, \& Luk, 2012 for a review). Executive functioning is an umbrella term describing a set of cognitive abilities, including inhibition of dominant responses, shifting between tasks or mental sets, as well as updating and monitoring working memory (Miyake et al., 2000). As bilinguals activate both languages even they only speak in one language at a time (e.g., Thierry $\& \mathrm{Wu}, 2007)$, they must selectively attend to the correct language while inhibiting the other competing language for effective communication in daily life. Earlier theories, such as Green (1998), have proposed that bilinguals' routine of inhibiting the irrelevant language during production can improve their inhibitory ability in non-verbal domains. For example, a number of studies have shown that bilinguals outperform monolinguals in a number of non-verbal conflict tasks, including the flanker task (Costa, Hernández, Sebastián-Gallés, 2008), the Simon task (Bialystok et al., 2004), and the Spatial-Stroop task (Bialystok, 2006).

Subsequent studies have extended research to other aspects of executive control components aside from inhibition. A major reason for this move was that researchers (e.g., Bialystok, 2006; Costa et al., 2008) not only found a bilingual advantage in the incongruent trials of the above conflict tasks, which require inhibitory control, but also in the congruent trials that do not require inhibition. Hence, researchers suggested bilinguals' cognitive advantage is not limited to inhibition ability, but is instead related to an enhancement in executive attention (Bialystok, 2017). Executive attention is the ability to control ones' attention, including ones' ability to maintain attention to the relevant part of the task and avoid directing attention to distractors. Bialystok (2017) has suggested that bilingual experience improves learners' attention systems as bilinguals regularly need to control their attention to accommodate two different language systems. For example, they need to differentiate between the two languages, switch attention between the two, and allocate their attention to the relevant language. Importantly, Bialystok (2015) has highlighted that bilingual experience not only enhances learners' executive function processing when they are selectively producing one of their languages, but also when they are selectively processing the two languages during comprehension. As such, infant bilinguals, who possess richer language comprehension than production, may also enjoy a cognitive advantage before the onset of a large productive vocabulary.

Indeed, some infant studies demonstrate that infant bilingualism may improve executive functioning. In a pioneering study, Kovacs and Mehler (2009a) examined whether Italian monolingual and Italian-other bilingual 7month-olds differ in cognitive control. Infants were 
conditioned to associations between a set of rules and visual rewards. These sets of rules could be conveyed auditorily or visually. For example, two rule structures were composed of three syllables, one with an AAB pattern (e.g. la-la-ga) and one with an ABA pattern (e.g. la-ga-la). In the experiment, infants were first trained to look at a toy (e.g., puppy A) at a particular visual location (e.g., right-side) after hearing one rule structure (e.g., AAB) and later were required to learn a new association (e.g., look at the puppy $\mathrm{B}$ on the left when hearing ABA). This task thus required infants to inhibit an earlier learned response and flexibly switch to a new reward response. Across three experiments that used either syllable or visual geometric shape rule patterns, the authors consistently found only bilingual infants successfully learned the new associations. In a similar study, Kovacs and Mehler (2009b) tested whether monolinguals and bilinguals of 12 months were able to simultaneously learn two different tri-syllabic structure rules: $\mathrm{AAB}$ and $\mathrm{ABA}$. They again associated each rule to a visual toy at a particular position on the screen (e.g. AABobject A-left side; ABA-object B-right side). At test, the researchers presented new tri-syllabic auditory stimuli that conveyed either an AAB or ABA structure. They then measured whether infants looked to the correct position after hearing the new auditory stimuli (e.g. AAB structure provokes looks to the left side). If infants looked at the correct corresponding position, it means they learned the corresponding structure rule. The researchers found that bilinguals were able to learn both rules, but monolinguals learned only one of the rule patterns (AAB but not ABA). To summarize, the two studies have suggested that bilingual infants may be better able to control interference and switch between two rule structures.

Over the past decade, however, only a few studies have attempted to replicate the studies in Kovacs and Mehler (2009a). For example, Ibánez-Lillo, Pons, Costa, \& Sebastián-Gallés (2010) discovered that both monolingual and bilingual infants of 8 months could inhibit the previously learned cue-reward pairing and successfully learn a new cue-reward pairing, suggesting no early bilingual advantage in cognition. In contrast, Pourllyaei and Byers-Heinlein (2018) found that 7-month-old bilinguals, but not monolinguals, were able to inhibit a previously learned cues' position and anticipate a newly learned cues' position. They specifically presented infants with a visualauditory cue (e.g., a colorful butterfly paired with a whistle sound) for the first 9 trials on one side of the screen (e.g., left) and then switched the position (e.g., right) of the visual-auditory cue for the next 9 trials. Further, Comishen, Bialystok and Adler (2019) also found that 6-month-old bilingual infants outperformed monolingual infants in a visual expectation cueing paradigm in which infants needed to change their anticipatory looks in response to the varying positions of the rewards. Together, the current evidence of bilingual infants' cognitive advantage is somewhat mixed and the literature is quite limited.
The current paper aims to address this research gap by attempting to replicate Kovacs and Mehler (2009a) in a different population of bilingual infants. Studying whether bilingual infants may have enhanced cognitive skills is a key research question, as it can inform researchers how a variety of language inputs in the early language environment (i.e., processing two language systems) may alter learners' cognitive and/or attention systems. However, we were not able to answer this question based on the current literature as there were only a few studies $(n=3$, two of which are unpublished) that have tried to replicate Kovacs and Mehler (2009a) and the limited findings are somewhat mixed. Given that a number of studies have failed to replicate bilingual cognitive advantages in adult participants (e.g., Kousaie \& Philips, 2012; Paap \& Greenberg, 2013), it is possible that the infant bilingual cognitive advantage is similarly not robust. Replication is a central focus in the current scientific community, with recent specific concerns about replicability in psychological science (Open Science Collaboration, 2015). Infant research is particularly vulnerable to the replication crisis as recruiting and testing infants is difficult, and researchers therefore normally report findings and draw conclusions from small samples (Frank et al., 2017). As such, the current paper contributes to the literature by testing whether bilingual infants would outperform monolingual infants in an experimental paradigm modified from Kovacs and Mehler (2009a) Experiment 3. The current experiment used geometric shapes to convey the abstract rule patterns. This would be a key test of the bilingual cognitive advantage, as researchers have argued that bilinguals' enhanced attention system should transfer to non-verbal cognitive tasks that require attention control (Bialystok, 2017).

There were several methodological differences between our experiment and those reported in Kovacs and Mehler (2009a). First, we tested 9.5-month-old infants, who were slightly older than those reported in Kovacs and Mehler (2009a). As mentioned above, Kovacs and Mehler (2009a) tested infants with abstract rule patterns (i.e., AAB/ABB structures) and paired these structures with different visual rewards at different locations of the screen. Some researchers have raised concerns of testing infants with abstract rules patterns (i.e., extracting patterns from stimuli) as this may quite challenging to participants this young (Comishen et al., 2019). Given that 7-month-old infants can learn some abstract rule patterns (Marcus et al., 1999) and Ibánez-Lillo et al (2010) reported that both monolingual and bilingual infants at 8 months can switch their responses when the cue-reward pairings had changed, we decided to test 9.5-month-old infants, who are a bit older than 8months, to ensure that the infants were sufficiently mature to handle the task demands. Furthermore, these slightly older infants would have even more bilingual experience, perhaps enhancing any effect of dual language exposure. Second, our abstract rule patterns were conveyed using both visual and auditory (non-linguistic) cues, as presenting 
information bimodally should facilitate infant abstract rule learning (Frank et al., 2009). Lastly, we reduced the number of trials during pre-switch and post-switch phases from nine to six. Our decision was based on pilot data (not included in this paper) that revealed 9.5-month-old infants lost interest in the screen after six to seven trials presenting the same abstract rule patterns during the pre-switch phase. Moreover, Kovacs and Mehler (2009a) have shown that 7month-old bilingual infants began switching their responses during post-switch phase from the fourth trial in Experiment 3. As we were testing older infants, we expected that older infants could switch their responses earlier in the postswitch phase. Thus, we set the number of trials in pre-switch and post-switch phases to six.

\section{Methods}

This experiment involved an anticipatory eye movement paradigm, modified from Experiment 3 in Kovács and Mehler (2009a). Infants were trained to predict the locations of visual rewards based on the structures of tone-shape sequences. The key manipulation was that the structure of the tone-shape sequence would change in the middle of the experiment. Successful learners must inhibit the previously learned tone-shape sequence and then learn the new one. Similar to others, we argue that this task measures infants' general cognitive skills, including working memory, attention and inhibitory control. First, infants need to use their working memory to process and track the information in the tone-shape sequences. Next, infants need to pay attention to the common structures across tone-shape sequences and the association between those structures and the locations of visual rewards. Lastly, as mentioned above, infants must rapidly inhibit the previously learned toneshape sequence structure in order to learn new tone-shape sequence structure during the post-switch phase.

\section{Participants}

Twenty-four monolingual English infants and 23 FrenchEnglish bilingual infants of 9.5 months were tested (Mean $=9.49$ months; S.D. $=0.64$ months), 22 female). All participants were living in a French-English city in an officially bilingual (French-English) country.

We used the Language Exposure Questionnaire (Bosch \& Sebástian-Gallés, 1997) to measure infants' language exposure to English and French. Infants were categorized as monolinguals if they had $90 \%$ or greater exposure to English. Infants were categorized as bilinguals if they had a minimum of $20 \%$ exposure to one language and a maximum of $80 \%$ exposure to the other language (Mean English exposure $=54.96 \%, \mathrm{SD}=15.64 \%$; Mean French exposure $=44.71 \%, \mathrm{SD}=15.77 \%)$. An additional four infants $(2$ monolinguals, 2 bilinguals) were tested but not included in the final analysis because of crying or fussiness.

\section{Stimuli}

Figure 1 illustrates sample stimuli and the procedure of the study. All stimuli were organized into 3 tone-shape sequences of two structures: $\mathrm{AAB}$ or $\mathrm{ABB}$. For $\mathrm{AAB}$ sequences, the first two tones and geometric shapes in the sequence were identical and the final pairing differed (e.g., circle-tone $\mathrm{C}$, circle-tone $\mathrm{C}$, star-tone $\mathrm{F}$ ). By contrast, for $\mathrm{ABB}$ sequence, the last two shape-tone pairings were identical and the first pairing differed (e.g., star-tone F, circle-tone $\mathrm{C}$, circle-tone $\mathrm{C}$ ).

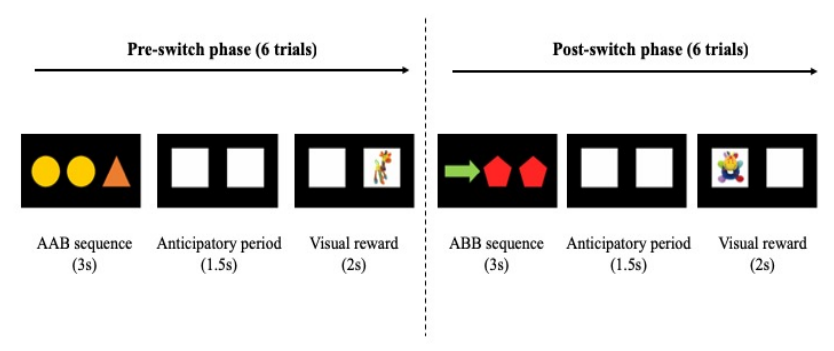

Figure 1. Sample visual stimuli and the procedure of the anticipatory eye movement paradigm.

The visual stimuli comprised six $\mathrm{AAB}$ shape sequences and six ABB sequences. Following the methodology in Kovács and Mehler (2009a), specific geometric shapes were used to generate the sequences. Three geometric shapes (arrow, circle, pentagon) were used for position A of the $\mathrm{AAB}$ and $\mathrm{ABB}$ sequences, whereas three other geometric shapes (moon, 5-pointed star, triangle) were used for position B. Each shape was presented in a different colour. For symmetrical geometric shapes (i.e., circle, pentagon, 5-pointed star and triangle), the size of each shape was $32 \mathrm{~cm} \mathrm{X} 32 \mathrm{~cm}$. For asymmetric geometric shapes (i.e., arrow and moon), the size of each shape was $34 \mathrm{~cm} \mathrm{X} 32 \mathrm{~cm}$. The shape sequences appeared on screen in the following manner. The first shape of the sequences was presented on the left side of the screen alone. Next, the second shape was added in the middle of the screen while the first remained onscreen. Finally, the third shape was added on the right side of the screen so that all three shapes appeared simultaneously on the screen for $3 \mathrm{sec}$. All shape sequences were displayed against a black background.

The audio stimuli were sequences of three musical tones. Two tone structures (i.e., AAB and ABB) were constructed to pair with the corresponding visual shape sequences. Three musical tones (A, D, E) could be paired with objects in position $A$, whereas three other musical tones $(C, F, G)$ could be paired with objects in position $B$.

The tone-shape sequence would be followed by a visual presentation of two white squares and the visual reward (see procedure for more details). The two white squares were presented side by side on the screen (each was $52.5 \mathrm{~cm}$ to $53.5 \mathrm{~cm}$ in size) for $1.5 \mathrm{sec}$. For the visual reward, one of two puppets (i.e., a giraffe or hippopotamus toy) appeared inside one of the two white squares on the left or right side of the screen. The puppet loomed from $20 \mathrm{~cm} \mathrm{X} 30 \mathrm{~cm}$ to 
$28 \mathrm{~cm} \mathrm{X} 49 \mathrm{~cm}$ in size for $2 \mathrm{sec}$. The presentation of puppets was accompanied by a chime sound.

\section{Apparatus and procedure}

Infants were seated on a parent's lap during the experiment. The parent wore headphones playing music with vocals to mask the sounds. The parent was instructed not to turn his/her head to the left-hand side or right-hand side. Instead, parent could either look at the infant or look at the center of the screen in order to minimize their influence of infants' attention to a particular side of the screen. At the beginning of each trial, we presented infants with attention-getting stimuli (e.g., an image of a baby and audio of a baby giggle). Once infants oriented their attention to the screen, the experimenter pressed a key to present the orientation stimuli to the infants. For the first trial, infants saw a video where a rotating ball changed its position from the left side to the right side of the screen. The ball first appeared on the left side and remained onscreen for $3.3 \mathrm{sec}$. The ball then reappeared on the right side of the screen for another $3.3 \mathrm{sec}$. The trial served to accustom infants with the experiment procedure where they would be trained to look at the left and right side of the screen to predict different visual rewards based on the tone-shape sequences.

After this orientation trial, there were two phases in the experiment: pre-switch and post-switch (see Figure 1). Each phase consisted of 6 trials. In the pre-switch phase, infants were trained that one tone-shape sequence structure (either $\mathrm{AAB}$ or $\mathrm{ABB}$ ) predicted a visual reward in a particular location (i.e., on the right or left of the screen). On each trial, infants were first presented with a tone-shape sequence (e.g., AAB) for $3 \mathrm{sec}$. The tone-shape sequence would then be replaced by two white squares on the screen for $1.5 \mathrm{sec}$. During this window of time, infants could make an anticipatory eye movement by directing their eye gaze to the square where the object would appear (anticipatory window period). Finally, a looming puppet (e.g., giraffe) would appear on one side of the screen (e.g., right side) for 2 sec. After presenting infants with 6 pre-switch trials, infants then entered a post-switch phase where a new structure of tone-shape sequences (e.g., ABB structure) predicted a different reward (e.g., hippopotamus) on a different location (e.g., left side). The procedure and length were identical to the pre-switch phase, aside from the differences above (i.e., sequence structure, visual reward type and location).

\section{Coding}

Following Kovács and Mehler (2009a), we coded infants' eye gaze during the anticipatory window period $(1.5 \mathrm{sec})$ for the dependent variable (DV). We coded infants' eye gazes to the right and left positions. We only counted eye gazes to the appropriate reward location as correct responses. Eye gazes to the opposite side of the reward location were all counted as incorrect. Videos were coded frame by frame (30 frames per second). Two trained undergraduate coders coded all trials independently and the reliability between their coding was high $(r=0.90, p<$ 0.0001 ). To obtain the proportion of correct anticipatory looks, we divided the number of frames looking at the correct location by the sum of total frames that infants looked at the correct and incorrect positions during anticipatory window period. For example, on a test trial, an infant looked at the correct position for a total of 20 frames and looked at the incorrect position for a total of 10 frames. The proportion of correct anticipatory looks would be 0.67 for this infant on this particular trial.

\section{Data analysis}

The DV was infants' proportion of correct anticipatory looks in each trial. Further, we expected that infants would increase the proportion of correct anticipatory looks over time, thus we tested whether the relationship between the DV and the number of trials (i.e., DV-trial slope) was positive or not. We also tested whether infants' language background would influence the DV-trial slope. For example, bilingual infants may have faster rate of learning the association between tone-shape sequence and the corresponding visual rewards. This would be reflected by a steeper DV-trial slope in the bilingual infant group. To model the variations of the DV and the DV-trial slopes in the experiment, we employed hierarchical linear modeling in our analyses. Because we have different hypotheses about infants' performance in the pre-switch and the postswitch phases. We ran two separate hierarchical linear models, one examined infants' performance in the preswitch phase (pre-switch model) and the other examined infants' performance in the post-switch phase (post-switch model). We predicted that infants' inhibitory control ability would only be reflected in their performance during the post-switch phase. As such, we expected that monolingual and bilingual infants would have similar proportion of correct anticipatory looks and DV-trial slopes during the pre-switch phase. By contrast, monolingual and bilingual infants would differ in terms of their proportion of correct anticipatory looks and DV-trial slopes during the postswitch phase. We used the lme4 package in R (Bates et al., 2015) to perform the hierarchical linear regression models. The regression models were fit by the restricted maximum likelihood approach and the $p$ values in the models were estimated by Satterthwaite approximations in the lmerTest package (Kuznetsova, Brockhoff, \& Christensen, 2016). The model was specified as follow: DV $\sim$ trial*language background $+(1+$ trial|Subject $)$ in the lme4 package.

\section{Results}

See Figure 2 for infants' performance across trials during the pre-switch and post-switch phases. In the pre-switch model, infants' language background $[\beta=0.047$, S.E. $=$ $0.066 p=0.47$ ] was not a significant predictor. We also 
found that the interaction between infants' language background and DV-trial slopes was not significant $[\beta=$ 0.020$, S.E. $=0.011, p=0.997]$, suggesting that the learning rate between monolingual and bilingual infants were similar in the pre-switch phase. The average DV-trial slope was significantly higher than zero across all participants $[\beta=$ $0.020, S . E .=0.076, p=0.012]$, suggesting that infants made more correct anticipatory looks over time. Finally, a onetailed $t$ test revealed that the average proportion of correct anticipatory looks in the pre-switch phase was significantly greater than the chance level $[M=0.59, \mathrm{t}(276)=4.909, \mathrm{p}<$ $0.001]$.

In the post-switch model, we also did not find a significant effect of infants' language background $[\beta=$ 0.015 , S.E. $=0.063, p=0.82]$. The interaction between infants' language background and DV-trial slopes was not significant $[\beta=-0.004$, S.E. $=0.023, p=0.878]$. This again indicates that the learning rate between monolingual and bilingual infants were similar in the post-switch phase. The average DV-trial slope was significantly higher than zero across all participants $[\beta=0.031$, S.E. $=0.012, p=0.009]$, suggesting that infants also showed a trend of improving their proportion of correct anticipatory looks over time during the post-switch phase. Finally, a one-tailed t test revealed that the average proportion of correct anticipatory looks in the post-switch phase was not significantly greater than the chance level $[M=0.45, t(268)=-2.63, p>0.99]$.

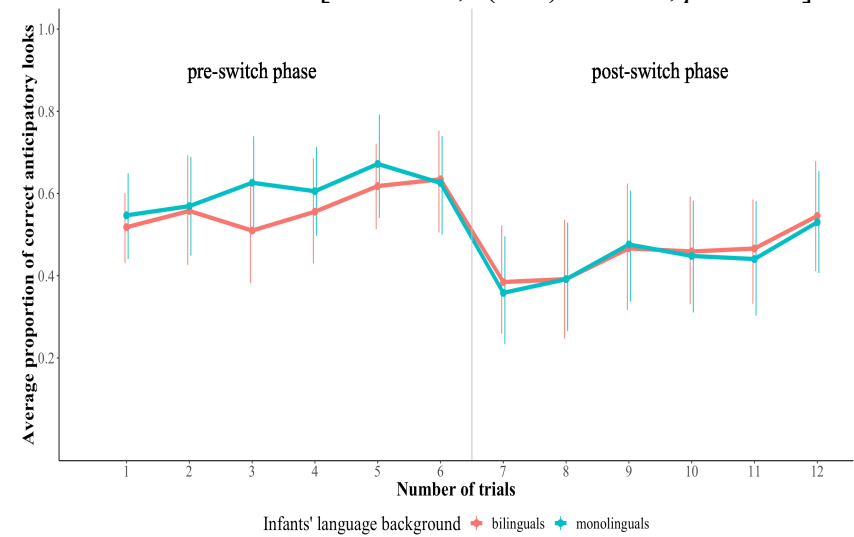

Figure 2. Infants' average proportion of correct anticipatory looks across trials during pre-switch and post-switch phases. Error bars indicate the $95 \%$ confidence intervals.

We did some follow up hierarchical linear regression models to further explore whether the degree of bilingual experience influences infants' performance. One aspect of bilingual experience is whether the bilingual infant has balanced exposure to the two languages. The degree of bilingualism was determined by the difference of percentage exposure between dominant language and nondominant language. For example, an infant with $60 \%$ exposure to English and 40\% exposure to French would get a score of 20, while an infant with 50\% exposure to English and French would get a score of 0 . Here, a smaller value in the degree of bilingualism reflected that the infant received a more balanced language exposure to French and English. The degree of bilingualism can be treated as a proxy for the variation of bilingual experience among our bilingual infants. In the following analyses, we only focused on bilingual infants and measured whether the degree of bilingualism contributes to the difference in infants' average proportion of correct anticipatory look during preswitch and post-switch phases. We specified two separate models for the pre-switch and post-switch phases. Each model was specified as DV trial*infants' degree of bilingualism $+(1+$ trial|Subject $)$ in the lme4 package. Across two models, we did not find any significant main effects and interaction between trials and bilingual infants' degree of bilingualism ( $\mathrm{ps}>0.30$ ). This suggested that bilingual experience (whether they hear more or less balanced exposure in daily environments) did not predict infants' performance in our cognitive task.

Finally, we also computed one-tailed t tests to examine whether infants' average proportion of correct anticipatory look in the last two trials during post-switch phase was significantly greater than chance level. The t test results were both non-significant in trial $11[M=0.45, t(40)=$ $0.95, p=0.827]$ and in trial $12[M=0.54, t(44)=0.79, p=$ $0.217]$, suggesting that infants' performance across the last two trials did not significantly differ from the chance level.

\section{General Discussion}

Our goal was to replicate Kovacs and Mehler (2009a) study and examine the potential positive effects of early bilingualism to infants' cognitive ability. We found that both monolingual and bilingual infants could extract the abstract rule patterns and associate the patterns to particular reward locations. Their performance generally improved as they made more correct anticipatory looks based on the abstract rule patterns over-time. However, we did not find evidence supporting that early bilingualism improves infants' inhibitory control, at least for the experiment tested here. In our study, we tested slightly older infants, who would have even more bilingual experience, and attempted to maximize the learning effects by presenting infants with bimodal visual-audio stimuli. Despite using these manipulations that may enhance any effect of bilingualism, we still found a null effect. Thus, our findings are consistent to recent studies (e.g., Kousaie \& Philips, 2012; Paap \& Greenberg, 2013) that bilingualism may not have a robust effect on learners' cognition.

Notwithstanding our efforts to maximize the learning effects in the current paradigm, it is important to note that infants' average proportion of correct anticipatory looks during the post-switch phase was not significantly above chance. This implies that infants generally found learning the associations between the tone-shape structure and the visual rewards in the post-switch phase more difficult than 
those in the pre-switch phase. Although 7-month-olds were previously reported to succeed in learning using this paradigm (Kovacs \& Mehler, 2009a), our results suggest that it may still be cognitively challenging for 9.5-montholds to learn the new association within the six trials of the post-switch phase. Future work is perhaps needed to explore a range of age-appropriate and simplified cognitive tasks to fully address the question of a correlation between cognitive ability and bilingualism in infancy.

Another possibility is that our null findings may be related to the language contexts in the bilingual infants' home/community. Byers-Heinlein, Morin-Lessard and Lew-Williams (2017) have suggested that bilingual infants' enhanced cognitive ability may be driven by exposure to language mixing contexts in their environments. Language mixing is prevalent in early bilingual environments where one/both parent(s) switch between two languages when speaking to their infants. Byers-Heinlein et al., (2017) have discovered that bilingual French-English infants demonstrated a switching processing cost when hearing sentences that alternated between two languages. The switching costs suggest that bilingual infants need to monitor and control their two languages when listening to speech that mixes languages. Thus, bilingual infants'

\section{References}

Bialystok, E. (2006). Effect of bilingualism and computer video game experience on the Simon task. Canadian Journal of Experimental Psychology, 60(1), 68-79.

Bialystok, E. (2015). Bilingualism and the development of executive function: The role of attention. Child Development Perspectives, 9(2), 117-121.

Bialystok, E. (2017). The Bilingual Adaptation: How Minds Accommodate Experience. Psychological Bulletin, 143, 233-262.

Bialystok, E., Craik, F. I. M., Klein, R., \& Viswanathan, M. (2004). Bilingualism, aging, and cognitive control: Evidence from the Simon task. Psychology and Aging, 19, 290-303.

Bialystok, E., Craik, F. I. M., \& Luk, G. (2012). Bilingualism: Consequences for mind and brain. Trends in Cognitive Sciences, 16, 240-250.

Bates, D., Mächler, M., Bolker, B., \& Walker, S. (2014). Fitting linear mixed-effects models using lme4. Journal of Statistical Software, 67, 1.

Bosch, L., \& Ramon-Casas, M. (2011). Variability in vowel production by bilingual speakers: Can input properties hinder the early stabilization of contrastive categories?. Journal of Phonetics, 39(4), 514-526.

Byers-Heinlein, K. (2013). Parental language mixing: Its measurement and the relation of mixed input to young bilingual children's vocabulary size. Bilingualism: Language and Cognition, 16(1), 32-48.

Byers-Heinlein, K., Morin-Lessard, E., \& Lew-Williams, C. (2017). Bilingual infants control their languages as enhanced cognitive skills may be a result of listening to mixed speech on a daily basis. Although the local area is quite bilingual, perhaps the specific bilingual infants in our study were not living in a home language environment where parents often mix their languages, thus minimizing their need of monitoring and switching between two language systems regularly. In the current paper, we did not collect relevant data to examine this possibility. Future work should explore how the degree of language mixing in early bilingual environment affects infants' cognitive ability.

In conclusion, we did not find support for bilingual cognitive advantages at 9.5 months, suggesting that advantages may not be robustly seen across different bilingual populations or different ages. However, we made note of other possible accounts for the replication failure, including how the bilingual environments and task demands of the current experiment matter. It is our hope that future work can address the existing research gap to further understanding of the effects of early bilingualism on infants' cognitive ability.

they listen. Proceedings of the National Academy of Sciences, 114, 9032-9037.

Comishen, K. J., Bialystok, E., \& Adler, S. A. (2019). The Impact of Bilingual Environments on Selective Attention in Infancy. Developmental science, e12797.

Costa, A., Hernández, M., \& Sebastián-Gallés, N. (2008). Bilingualism aids conflict resolution: Evidence from the ANT task. Cognition, 106, 59-86.

Fennell, C. T., Tsui, A. S. M., \& Hudon, T. M. (2016). Speech perception in simultaneously bilingual infants. In S. Montanari \& E. Nicoladis (Eds.) Bilingualism across the lifespan: Factors moderating language proficiency. Washington, USA: American Psychological Association.

Frank, M. C., Bergelson, E., Bergmann, C., Cristia, A., Floccia, C., Gervain, J., ... \& Lew-Williams, C. (2017). A collaborative approach to infant research: Promoting reproducibility, best practices, and theory-building. Infancy, 22(4), 421-435.

Frank, M. C., Slemmer, J. A., Marcus, G. F., \& Johnson, S. P. (2009). Information from multiple modalities helps 5-month-olds learn abstract rules. Developmental Science, 12(4), 504-509.

Ferjan Ramìrez, N., Ramìrez, R. R., Clarke, M., Taulu, S., \& Kuhl, P. K. (2017). Speech Discrimination in 11Month-Old Bilingual and Monolingual Infants: A Magnetoencephalography Study. Developmental Science, 20(1), e12427.

Green, D. W. (1998). Mental control of the bilingual lexico-semantic system. Bilingualism: Language and Cognition, 1, 67-81. 
Ibánez-Lillo, A., Pons, F., Costa, A., \& Sebastián-Gallés, N. (2010). Inhibitory control in 8-month-old monolingual and bilingual infants: Evidence from an anticipatory eye movement task. Poster presented at the 22nd Biennial International Conference on Infant Studies, Baltimore, MD.

Kovács, Á. M., \& Mehler, J. (2009a). Cognitive gains in 7month-old bilingual infants. Proceedings of the National Academy of Sciences, 106(16), 6556-6560.

Kovács, Á. M., \& Mehler, J. (2009b). Flexible learning of multiple speech structures in bilingual infants. Science, 325(5940), 611-612.

Kousaie, S., \& Phillips, N. A. (2012). Conflict monitoring and resolution: Are two languages better than one? Evidence from reaction time and event-related brain potentials. Brain Research, 1446, 71-90.

Kuznetsova, A., Brockhoff, P.B., Christensen, R. H. B. (2016). lmerTest: Tests in Linear Mixed Effects Models. $\mathrm{R}$ package version 2.0-29. Retrieved from: http://CRAN.R-project.org/package=lmerTest.

Marcus, G. F., Vijayan, S., Rao, S. B., \& Vishton, P. M. (1999). Rule learning by seven-month-old infants. Science, 283(5398), 77-80

Miyake, A., Friedman, N. P., Emerson, M. J., Witzki, A. H., Howerter, A., \& Wager, T. D. (2000). The unity and diversity of executive functions and their contributions to complex "frontal lobe" tasks: A latent variable analysis. Cognitive Psychology, 41, 49-100.

Open Science Collaboration. (2015). Estimating the reproducibility of psychological science. Science, 349.

Paap, K. R., \& Greenberg, Z. I. (2013). There is no coherent evidence for a bilingual advantage in executive processing. Cognitive Psychology, 66(2), 232-258.

Pour Ilyaei, S., \& Byers-Heinlein, K. (2018, July). Cognitive capacity in infancy: How is it linked to bilingualism? Poster presented at the International Congress on Infant Studies, Philadelphia, PA.

Thierry, G., \& Wu, Y. J. (2007). Brain potentials reveal unconscious translation during foreign language comprehension. Proceedings of the National Academy of Sciences of the United States of America, 104, 1253012535. 